\title{
Survey of the awareness of xerostomia in elderly
}

\author{
Nur Adlina Diyana Md Ismail*, Elizabeth Fitriana*, Sri Tjahajawati** \\ *Department of Oral Medicine Faculty of Dentistry Universitas Padjadjaran, Indonesia \\ **Department of Oral Biology Faculty of Dentistry Universitas Padjadjaran, Indonesia
}

\section{ABSTRACT}

Introduction: One of the significant public health issues in this modern time is the rapidly escalating elderly popula-tion. The number of people older than 65 years will double to $14 \%$ of the world's population in the next 30 years. Due to various chronic conditions as well as prescription medications, it is presumed that more than $30 \%$ of the population aged 65 years and older is experiencing xerostomia, a condition not well known among the elderly. This study was not focusing on the disease per se but aimed to evaluate the awareness of the condition. Methods: The method of this study was descriptive with the survey method. Questionnaires were given to a sample gathered consecutively in the period of one month. The results were then presented in table form. Results: Out of the 63 respondents, $15.9 \%$ were considered to have full awareness of this condition. $44.4 \%$ of respondents were in the moderate awareness category, and $39.7 \%$ were not aware of the disease. Conclusion: Majority of the elderly have a moderate level of awareness towards xerostomia.

Keywords: Awareness, xerostomia, elderly.

\section{INTRODUCTION}

In recent years, the world has witnessed its elderly population increase in number significantly. The number of people older than 65 will double to $14 \mathrm{from} 7 \%$ of the world's population in the next 30 years, rising to 1.4 billion by 2040 from about 506 million in the middle of $2007 .^{1}$

Even with significant advancement in the medical field, the increase in ageing population poses a severe problem medically. The Global Burden of Disease predicts a substantial increase in disability caused by increases in age-related chronic disease in all regions of the world. ${ }^{2}$ All of these acute and chronic conditions have the potential oral sequel, particularly in the older and more medically compromised adult. ${ }^{3}$ A situation that is closely related to dentistry and the elderly is xerostomia. It is presumed that more than $30 \%$ of the population aged 65 years and older are experiencing xerostomia. ${ }^{4}$

Ageing per se is not associated with xerostomia, although the use of prescription medications is strongly associated with dry mouth, with the polypharmacy characteristic of older adults being a leading cause. ${ }^{5}$ Relationship between xerostomia and well-being has systematically been investigated using different health-related quality of life scales, and the studies indicate a correlation between the quality of life and oral health among individuals with xerostomia.

A study done by Folke et al. ${ }^{6}$ found extensive findings that relate xerostomia with a devastating and debilitating impact on multiple domains of 
Survey of the awareness of xerostomia in elderly (Nur Adlina Diyana Md Ismail et al.)

well-being. During the past decade, xerostomia has received increased attention as it affects important aspects of oral tissues and essential oral functions. ${ }^{6}$ Previously, a study has been done to explore and describe views of xerostomia among health care professionals. It was found that xerostomia, although a well-known problem, there is inadequate management of patients with xerostomia. Xerostomia is also underestimated and an ignored issue. ${ }^{6,7}$ However, there has not been any review done on whether the patient is aware of this condition.

Potential problem that could affect the oral cavity includes susceptibility to caries that in turn would create a domino effect that will eventually cause the loss of dentition. Xerostomia affects the quality of life, but it has been considered a neglected condition of the oral cavity. Therefore, this study was aimed to evaluate the awareness of xerostomia in the elderly, with the hope that with this study, the condition of xerostomia will be paid more attention.

\section{METHODS}

The research was a descriptive survey. A series of questions in the form of a questionnaire was given out to the sample. The population of this research were patients that come to the Geriatrics Polyclinic of Hasan Sadikin Hospital. The sampling method used was consecutive sampling, where every patient that fits into the inclusion and exclusion criteria was given the questionnaire.

The inclusion criteria were men and women above the age of 60 years old, experiencing xerostomia, able to give informed consent, and able to speak, read, write and communicate in Bahasa Indonesia. Exclusion criteria are those who have a significant medical illness with unstable medical conditions which could lead to difficulty in answering the questionnaire.

The questionnaire used in this study was the Questionnaire on Awareness of Xerostomia in Elderly Based on the Definition of Awareness by Collins Thesaurus of English Language. Awareness was defined as having knowledge, understanding and appreciation. This definition was reflected in the questionnaire consisted of 5 questions per section (knowledge, understanding, appreciation) with an overall total of 15 questions. The questions are limited to 5 questions per section, keeping in mind that the respondents were elderly, and it was not the intention of this study to burden them. Before being handed out, the questionnaires were given out to 15 respondents to assess the validity. Each of correct answer was given 1 point and 0 point for false answer. The points were added to get the cumulative score. Where $\mathrm{x}=$ cumulative points from knowledge section; $y=$ cumulative points from understanding section; $z=$ cumulative points from appreciation section. From the cumulative points from all parts, the level of awareness was grouped into fully aware, quite aware, and unaware. The range of cumulative points was 4 . It was calculated by the formula, where maximum score $=13$; minimum score $=1$; and the number of category $=3$.

$$
\text { Interval } \frac{=\text { Maximum Score }- \text { Minimum Score }}{\text { Number of Category }}
$$

Table 1. Scoring for level of awareness

\begin{tabular}{ll}
\hline Level of awareness & Cumulative points \\
\hline Fully aware & $10-13$ \\
Quite aware & $6-9$ \\
Unaware & $1-5$ \\
\hline
\end{tabular}

\section{RESULTS}

Results of this study are presented in table form. Each section is tabulated in the form of correct and false answers for all respondents and the number of respondents according to the level of awareness.

Table 2 Frequency and percentage average of correct and false answer for knowledge section

\begin{tabular}{lll}
\hline & Correct & False \\
\hline Frequency & 107 & 208 \\
Percentage average & $34.0 \%$ & $66.0 \%$ \\
\hline
\end{tabular}

Table 3 Frequency and percentage average of correct and false answer for understanding section

\begin{tabular}{lll}
\hline & Correct & False \\
\hline Frequency & 214 & 101 \\
Percentage average & $67.9 \%$ & $32.1 \%$ \\
\hline
\end{tabular}


Table 4. Frequency and percentage average of correct and false answer for appreciation section

\begin{tabular}{lll}
\hline & Correct & False \\
\hline Frequency & 100 & 215 \\
Percentage average & $31.7 \%$ & $68.3 \%$ \\
\hline
\end{tabular}

Table 5. Number of respondents towards level of awareness

\begin{tabular}{lllll}
\hline $\begin{array}{c}\text { Range of } \\
\text { marks }\end{array}$ & \multicolumn{1}{c}{$\begin{array}{c}1-5 \\
\text { (Unaware) }\end{array}$} & $\begin{array}{c}\text { 6-9 (Quite } \\
\text { aware) }\end{array}$ & $\begin{array}{c}10-13 \text { (Fully } \\
\text { aware) }\end{array}$ & \\
\hline Respondents & 24 & 28 & 11 & $\sum=63$ \\
Percentage & $39.7 \%$ & $44.4 \%$ & $15.9 \%$ & $\sum=100$ \\
\hline
\end{tabular}

\section{DISCUSSION}

From the results can be seen that the percentage average of the correct answer for the knowledge section was $34.0 \%$ which much lower than the percentage average of the correct answer for the understanding section which accounts for $67.9 \%$.

The knowledge section with the question regarding the main basics of the condition xerostomia was having a meagre percentage average of the correct answer. This could be due to the lack of information given to the general public or more importantly, the elderly. In the United States, the insufficient exposure on xerostomia is tackled by increasing the promotion and public health awareness on this condition with an effort by both the American Dental Association and American Pharmacy Association. ${ }^{8}$ Health education is a part of health promotion. It is defined as the combination of planned learning experiences to facilitate voluntary actions conducive to health. ${ }^{9}$ Therefore by promoting about xerostomia, the basic knowledge on this condition can be improved as well as empowering the elderly to take a more conducive action in dealing with xerostomia.

The results show that the understanding section has a more percentage average of a correct answer than knowledge section. It is interesting that while having little or no knowledge of the condition, the understanding section has a considerably high percentage average of the correct answer. This could be explained with the Self-Regulation Theory described by Leventhal. This theory postulates that illness representation will lead the individual to engage in common- sense health behaviours to cope with a particular illness. For example, in this case, drinking water is the logical thing to do to relieve dry mouth even if the respondents have never known the condition xerostomia as asked in the knowledge section. ${ }^{10}$ This was further proved by a study done in the University of Granada that states patients are not experts, and their ideas and perception of their disease are based on their own experience, self-knowledge and other sources. The idea that patients have of their condition affects their own coping and adaptation to it. ${ }^{11}$

The appreciation section has an overwhelmingly false answer with $68.3 \%$ as the percentage average of the false answer. The question of whether or not they use any aid to relieve dry mouth has the most negative answer. This could be due to the low availability lack of promotion of aids for xerostomia such as artificial saliva. For the question of whether or not they increase their water intake had the highest correct answer. This could be explained by the Self-Regulation Theory, as mentioned earlier.

\section{REFERENCES}

1. Waters R. Elderly to Double to $14 \%$ of Global Population by 2040 (Update2) Bloomberg News. 2007.

2. Murray CJ, Lopez AD, World Health Organization. The global burden of disease: a comprehensive assessment of mortality and disability from diseases, injuries, and risk factors in 1990 and projected to 2020: summary. World Health Organization; 1996.

3. National Institutes of Health: National Institute on Aging. Why population aging matters; A global perspective. Bethesda; 2007. p. 2.

4. Rehiman AM, Shankar Redd YU, Reddy R. Geriatrics and Oral Health. Dentaires Revista.2010;2(1):29-34.

5. Van der Putten GJ, Brand HS, Schols JMG, de Baat $C$. The diagnostic suitability of a xerostomia questionnaire and the association between xerostomia, hyposalivation and medication use in a group of nursing home residents. Clin Oral Invest. 2010;5:185-92.

6. Quandt S, Savoca M, Leng X, Chen H, Bell A, Gilbert $G$ et al. Dry mouth and dietary quality in older adults in north Carolina. J Am Geriatr 
Soc. 2011;59(3):439-445.

7. Folke $S$, Paulsson $G$, Fridlund B, Soderfeldt $B$. The subjective meaning of xerostomiaan aggravating misery. Internat J Qualitative Studies on Health and Well-being. 2009;4(4).

8. Folke S, Fridlund B, Paulsson G. Views of xerostomia among health care professionals: a qualitative study. Journal of clinical nursing. 2009;18(6):791-8.

9. American Dental Association. Dentists, Pharmacists Raise Awareness of MedicationInduced Dry Mouth. ADA Press Release. 2011. Available from: http://www. ada.org/6114.aspx

10. Detels R, McEwen J, Beaglehole R, Tanaka H. Oxford Textbook of Public Health: The Scope of Public Health. New York. Oxford University Press; 2002. p. 124-5.

11. DiefenbachMA, LeventhalH. Thecommon-sense model of illness representation: Theoretical and practical considerations. J Social Distress and the Homeless. 1996;5(1):11-38.

12. De los Santos Roig M. The impact of chronic diseases on patients also depends on their perception of the disease. University of Granada. 2010. 